\title{
SŷntheticBiologialtech Library
}

Subscriber access provided by Caltech Library

\section{Letter}

\section{Assessment of Robustness to Temperature in a Negative Feedback Loop and a Feedforward Loop}

Abhilash Patel, Richard M. Murray, and Shaunak Sen

ACS Synth. Biol., Just Accepted Manuscript • DOI: 10.1021/acssynbio.0c00023 • Publication Date (Web): 11 Jun 2020

Downloaded from pubs.acs.org on June 12, 2020

\section{Just Accepted}

"Just Accepted" manuscripts have been peer-reviewed and accepted for publication. They are posted online prior to technical editing, formatting for publication and author proofing. The American Chemical Society provides "Just Accepted" as a service to the research community to expedite the dissemination of scientific material as soon as possible after acceptance. "Just Accepted" manuscripts appear in full in PDF format accompanied by an HTML abstract. "Just Accepted" manuscripts have been fully peer reviewed, but should not be considered the official version of record. They are citable by the Digital Object Identifier (DOI@). "Just Accepted" is an optional service offered to authors. Therefore, the "Just Accepted" Web site may not include all articles that will be published in the journal. After a manuscript is technically edited and formatted, it will be removed from the "Just Accepted" Web site and published as an ASAP article. Note that technical editing may introduce minor changes to the manuscript text and/or graphics which could affect content, and all legal disclaimers and ethical guidelines that apply to the journal pertain. ACS cannot be held responsible for errors or consequences arising from the use of information contained in these "Just Accepted" manuscripts. 


\title{
Assessment of Robustness to Temperature in a Negative Feedback Loop and a Feedforward Loop
}

\author{
Abhilash Patel, ${ }^{\dagger}$ Richard M. Murray, ${ }^{\ddagger}$ and Shaunak Sen ${ }^{*, \dagger}$ \\ $\dagger$ Department of Electrical Engineering, Indian Institute of Technology Delhi, Hauz Khas, \\ New Delhi 110016, INDIA \\ $\ddagger$ California Institute of Technology, Pasadena, CA 91125, USA \\ E-mail: shaunak.sen@ee.iitd.ac.in
}

\begin{abstract}
Robustness to temperature variation is an important specification in biomolecular circuit design. While the cancellation of parametric temperature dependencies has been shown to improve the temperature robustness of the period in a synthetic oscillator design, the performance of other biomolecular circuit designs in different temperature conditions is relatively unclear. Using a combination of experimental measurements and mathematical models, we assessed the temperature robustness of two biomolecular circuit motifs - a negative feedback loop and a feedforward loop. We found that the measured responses of both the circuits changed with temperature, both in the amplitude and in the transient response. We also found that, in addition to the cancellation of parametric temperature dependencies, certain parameter regimes could facilitate the temperature robustness of the negative feedback loop, although at a performance cost. We discuss these parameter regimes in the context of the measured data for the negative feedback loop. These results should help develop a framework for assessing and


designing temperature robustness in biomolecular circuits.

\section{Keywords}

synthetic biology, temperature robustness, negative feedback, feedforward loop

An important specification for design is robust functionality in different environmental conditions. Temperature is a global environmental variable that can affect performance in multiple design contexts. Robustness to temperature change is an important specification in contexts such as semiconductor electronics ${ }^{1}$ and instrumentation ${ }^{2}$. This temperature dependence arises because the underlying components, such as the current-voltage characteristic in semiconductors, are temperature-dependent. A standard way to engineer temperature robustness is to design and configure component temperature dependencies in such a way that they cancel each other out. Since the properties of biological design substrates such as DNA, RNA, and proteins can also be temperature-dependent, robustness to temperature changes is an important consideration for design in biology as well.

Robustness of biomolecular circuit function to temperature variations is an important problem in both natural and synthetic contexts (Fig. 1) ${ }^{3,4}$. For example, in circadian rhythms, the mechanisms underlying the independence of the period to temperature changes have been investigated ${ }^{5,6}$. In another instance, the robustness of bacterial chemotaxis to temperature variations has been examined ${ }^{7}$. In both these contexts, the common principle for temperature robustness is to have "matched" parametric temperature dependencies, meaning that the temperature dependence of the circuit parameters is such that they cancel each other out. This principle was generalized using metabolic control analysis ${ }^{8}$. Synthetic oscillators can also have temperature-dependent periods ${ }^{9}$; relatively recently, a temperaturesensitive mutant transcription factor was used to compensate for the effect of temperature ${ }^{10}$. 
We investigated, primarily by computation, the propagation of temperature dependence in simple biomolecular circuit models, and noted that in addition to the matching temperature dependence of parameters, certain parameter regimes could also impart temperature robustness ${ }^{11,12}$. More recently, a multiscale approach was used to explain the effects of temperature on simple negative and positive feedback circuits in yeast ${ }^{13}$. These case studies highlight some principles and frameworks that can facilitate the design of temperature robustness in biomolecular circuits.

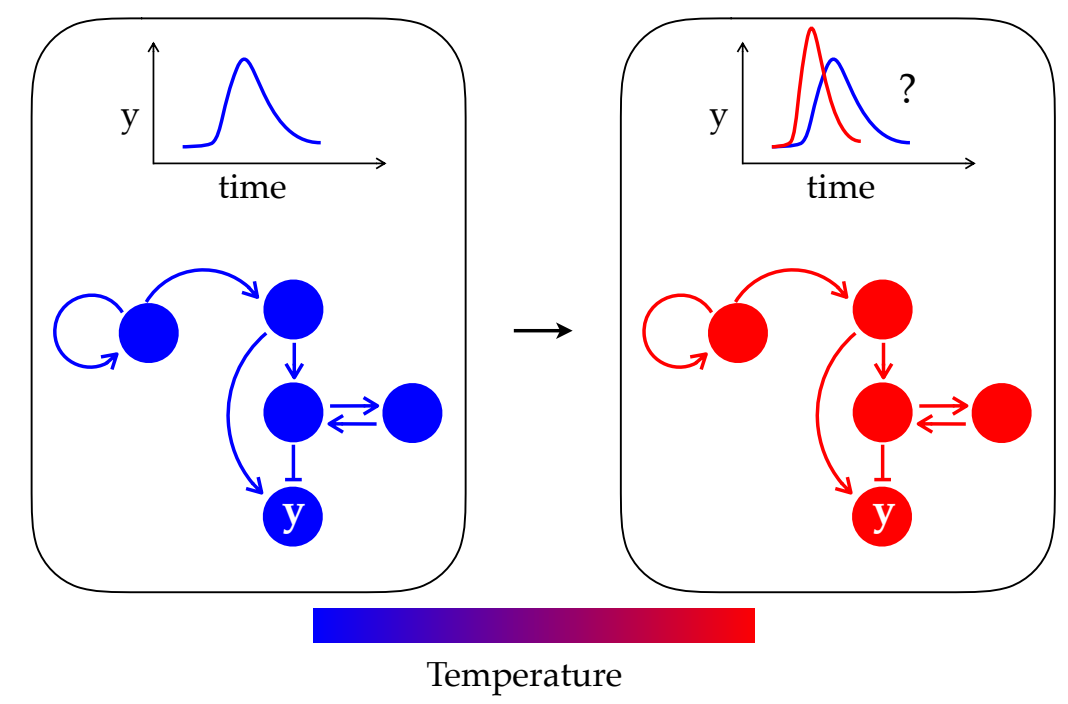

Figure 1: Biomolecular circuit response may change with temperature. Arrows represent interactions between the circle-shaped biomolecules. Blue and red represent cold and hot temperatures, respectively.

There are three notable aspects relating to temperature robustness in biomolecular circuits. The first is the similarity between the biomolecular contexts and other engineering contexts in achieving temperature robustness, through a suitable matching of the parametric temperature dependencies. The second is the relative ease with which biomolecular circuit outputs can be measured in comparison with the biomolecular circuit parameters, making the design of such matching temperature dependencies a matter of finding the right mutant, if it exists. The third is the possibility that some parameter regimes of behavior can facilitate temperature robustness. A characterization of the temperature robustness of standard 
biomolecular circuit designs may help in facilitating the design of temperature robustness.

Here, we investigated the extent of robustness to temperature changes in two biomolecular circuit motifs - a negative feedback loop and a feedforward loop. For this, we used a combination of experimental measurements and mathematical models. We found that the responses of these circuits changed with temperature, both in the amplitude and in the transient response. We analyzed the underlying mathematical models, and found that along with the parameters having matched temperature dependencies, specific parameter regimes could also facilitate temperature robustness, although at a performance cost. For the negative feedback circuit, in particular, a strong negative feedback could be more robust to temperature changes than a weaker one, but with lower expression levels. These results may help develop a framework to assess and design robustness to temperature in biomolecular circuits.

\section{Results and Discussion}

Experimental Assessment of Temperature Dependence. We experimentally assessed the temperature dependence of two circuits - a previously investigated negative feedback loop ${ }^{14}$ and a previously constructed feedforward loop realization (Addgene plasmid \#45789). Both of these belong to the set of circuits that have been identified as motifs in genetic networks ${ }^{15}$.

The negative feedback circuit used has the transcriptional repressor TetR expressed under the $P_{\text {tet }}$ promoter (Fig. 2a; Methods). TetR represses the $P_{\text {tet }}$ promoter, thus forming the negative feedback loop. The TetR protein is fused to a green fluorescent protein (GFP). The inducer anhydrotetracycline (aTc) can tune the strength of the negative feedback through its inhibitory effect on the transcriptional activity of TetR.

We measured the response of the circuit at $29{ }^{\circ} \mathrm{C}$ and $37{ }^{\circ} \mathrm{C}$ (Fig. 2b-d; Methods). These measurements were done at different concentrations of aTc. We found that the optical 

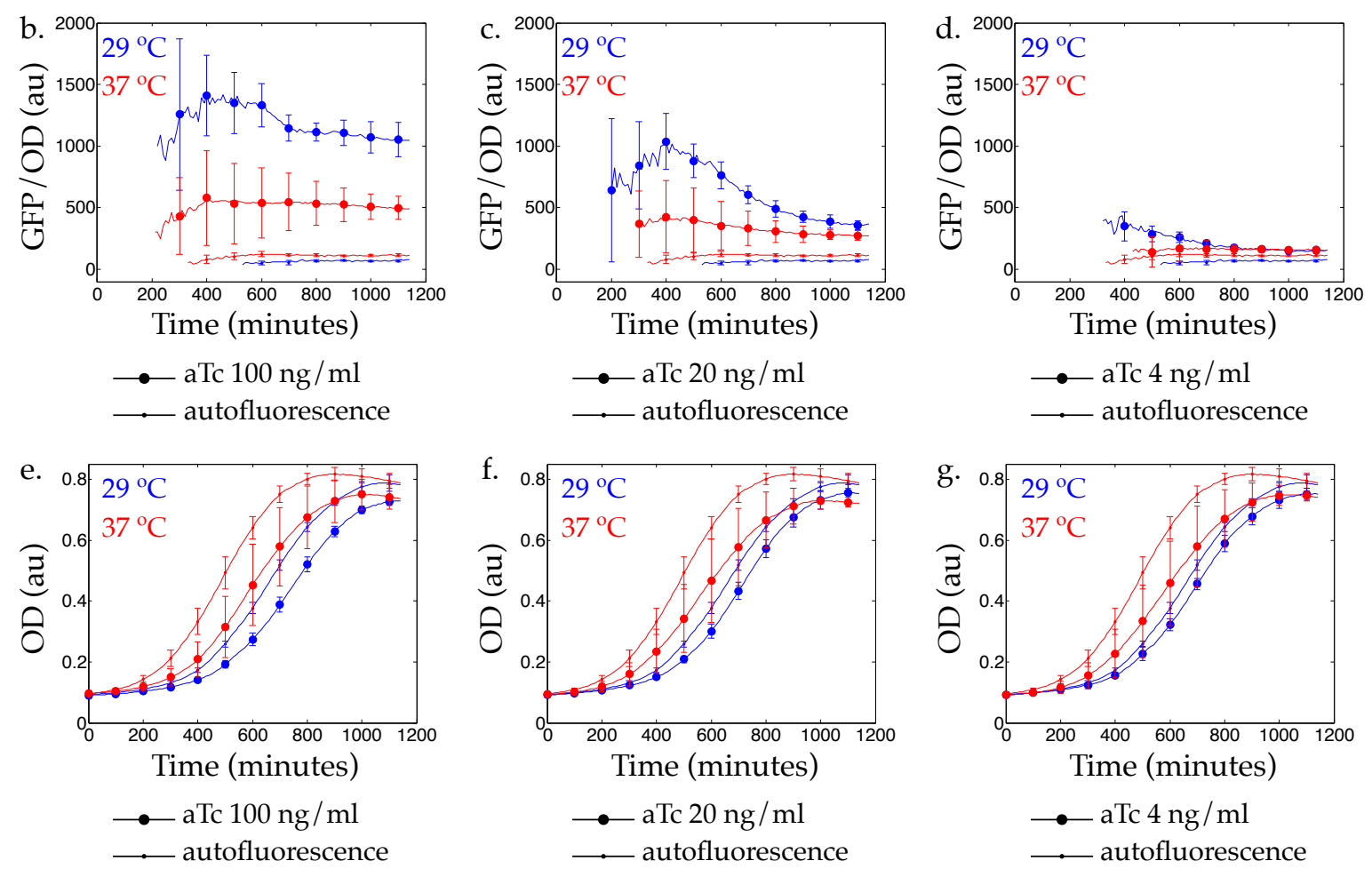

Figure 2: Response of a negative feedback loop depended on temperature. a. Schematic of the circuit. b. Blue and red lines are the responses measured at $29{ }^{\circ} \mathrm{C}$ and $37^{\circ} \mathrm{C}$, respectively. Circles and dots indicate the circuit response and the autofluorescence, respectively. The aTc concentration was $100 \mathrm{ng} / \mathrm{ml}$ and it was added at Time $=0$. c. Same as in b., with an aTc concentration of $20 \mathrm{ng} / \mathrm{ml}$. d. Same as in b., with an aTc concentration of $4 \mathrm{ng} / \mathrm{ml}$. e., f., and g. are the optical density measurements for the responses in b., c., and d., respectively.

The feedforward loop circuit used consists of the transcriptional activator AraC, the transcriptional repressor TetR, and a degradation-tagged green fluorescent protein GFPssrA (Fig. 3a; Methods). AraC is constitutively expressed and a transcriptional activator 
in the presence of the inducer arabinose. TetR is expressed from $P_{B A D}$, an AraC-activated promoter. GFP-ssrA is expressed from a modified $P_{B A D}$ promoter with TetR operator sites so that it is repressible by TetR.
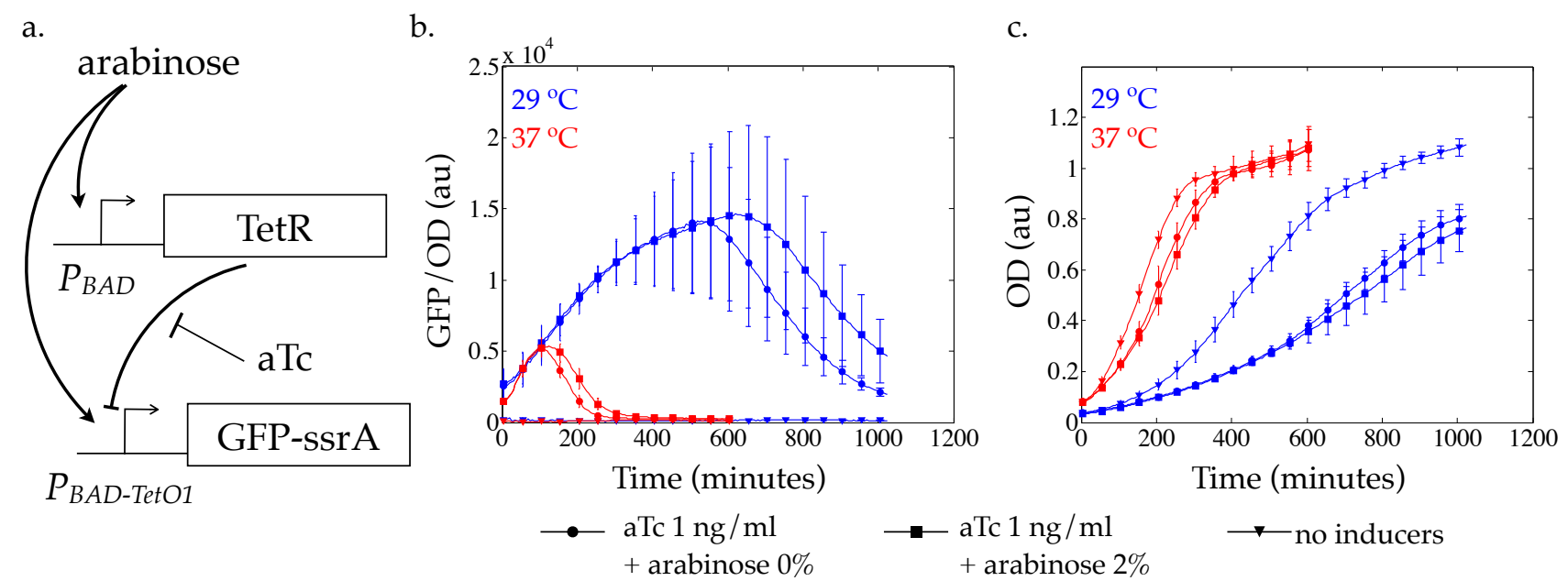

Figure 3: Response of a feedforward loop depended on temperature. a. Schematic of the circuit. b. Blue and red lines are the responses measured at $29{ }^{\circ} \mathrm{C}$ and $37{ }^{\circ} \mathrm{C}$, respectively. Circles, squares, and triangles are the responses for the indicated inducer conditions. Arabinose was added at Time $=0$ and aTc was added at Time $=-120$ minutes. c. Optical density measurements corresponding to b.

We measured the response of the circuit at $29{ }^{\circ} \mathrm{C}$ and $37^{\circ} \mathrm{C}$ and found that the optical density-normalized fluorescence showed a pulse response at both the temperatures (Fig. 3b; Methods). While the circuit was designed to generate a pulse in GFP-ssrA expression in response to the addition of arabinose, a fixed level of aTc was required for the pulse to be observed, perhaps due to the dominant effect of TetR. In the presence of aTc, the addition of arabinose had a small effect on increasing the amplitude of the pulse. The properties of the observed pulse response differed with the change in temperature, both in the amplitude of the pulse as well as in the time of its peak response.

These experimental measurements showed that the overall response of the two recurring circuits depended on temperature. This temperature dependence and its extent could have been determined by multiple factors. These included the temperature dependence observed 
in the optical-density-based growth curves of the two circuits (Fig. 2e-g, Fig. 3c), with slower growth corresponding to higher amplitudes, as well as the strength of the feedback (Fig. 2b-d), with a stronger feedback (lower aTc) corresponding to a smaller difference across the temperatures as well as a low overall amplitude.

Computational Assessment of Temperature Dependence. To complement the above experimental assessment, we considered simple models of these circuits to assess the effect of temperature on the circuit response.

For the negative feedback circuit, we considered the standard model of a protein $\mathrm{X}$ that transcriptionally represses its own expression and is degraded in a first-order process ${ }^{14}$,

$$
\frac{d x}{d t}=\frac{\alpha}{1+x / k}-\gamma x
$$

where $x$ is the protein level, $\alpha$ is the maximal production rate, $k$ is the DNA binding constant of $\mathrm{X}$ to its own promoter, and $\gamma$ is the degradation rate constant. The nominal values of the parameters were chosen as follows: $\alpha=100 \mathrm{nM} / \mathrm{hr}, k=10 \mathrm{nM}$, and $\gamma=1 / \mathrm{hr}$. These parameters were chosen to indicate the scale of the values and as a starting point for exploring the parameter space further. The parameter $\gamma$ was set primarily by the rate of growth of E. coli, which can double in at least 30 minutes $^{16}$. The corresponding timescale of $\ln 2 / 30$ /minute was taken as 1 / hr. A previously considered steady state level was 200 molecules/ cell $^{14}$ and in this context, 1 molecule/ cell $\sim 1 \mathrm{nM}^{17,18}$. Because $\max _{x \geq 0} \alpha /(1+x / k)=\alpha$ the maximum steady state value possible was $\alpha / \gamma$ (Supplementary Fig. 5). So, we chose $\alpha=100 \mathrm{nM} / \mathrm{hr}$ for the above value of $\gamma$. Further, a previously considered value of $k$ was $10 \mathrm{nM}^{14}$.

As a reference, we also considered a model without feedback,

$$
\frac{d x}{d t}=\alpha-\gamma x
$$

which can be exactly solved, $x(t)=\frac{\alpha}{\gamma}\left(1-e^{-\gamma t}\right)$, for a zero initial value of $x$. 
To assess the temperature dependence of the response, we used random sampling of the parameter space as a mathematical tool to assume the temperature dependencies of the parameters and compute the relative change in the steady state and the transient response. We modeled the parametric temperature dependencies based on the temperature coefficient $Q_{10}$, the amount that each parameter is scaled with when the temperature increases by 10 ${ }^{\circ} \mathrm{C}$. A $Q_{10}$ of 1 corresponds to perfect temperature robustness where there is no change with temperature, while a very large or a very small $Q_{10}$ signifies extreme temperature sensitivity. Since typical values of the $Q_{10}$ are in the range $2-3^{6}$, we assumed the $Q_{10}$ 's of $\alpha$ and $\gamma$ to be lying within this range. The parameter $k$ was a ratio of the dissociation and association rates for the binding of the transcriptional repressor $\mathrm{X}$ with its own promoter, each of which may have a $Q_{10}$ in the range 2-3. Assuming that the numerator and denominator in this ratio vary independently, the $Q_{10}$ of $k$ was considered to be in the range $0.66-1.5$. We computed the steady states and the transient responses for $M=100$ different parameter sets in the neighbourhood of the nominal parameter set (Supplementary Fig. 6). We chose these parameter sets by multiplying each nominal parameter value by $10^{r}$, where $r$ was a uniform random variable in the range $(-1,1)$. For each parameter set, we generated $N=100$ sets of $Q_{10}$ values for each parameter and computed the steady state and the transient response after scaling each parameter by its $Q_{10}$ value. The $Q_{10}$ values were chosen randomly from their respective ranges in a uniform fashion (Fig. 4a, d). The histograms of the temperature coefficients of the steady state $\left(Q_{x s s}\right)$, the ratio of the steady state obtained after scaling each parameter with its $Q_{10}$ value and the steady state value before scaling, are shown in Fig. 4b, e. Recall that a $Q_{10}$ of exactly one corresponds to perfect temperature robustness wherein there is no change with temperature. For the model without feedback, the mean was 1.01 and the standard deviation was 0.17 . For the negative feedback model, the mean was 1.03 and the standard deviation was 0.14 . These computations could be better understood 
using the model without feedback. The steady state in this model was $x_{s s}^{T}=\alpha^{T} / \gamma^{T}$ and

$$
Q_{x s s}=\frac{x_{s s}^{T+10}}{x_{s s}^{T}}=\frac{\alpha^{T+10} / \gamma^{T+10}}{\alpha^{T} / \gamma^{T}}=\frac{Q_{\alpha}}{Q_{\gamma}} .
$$

For the chosen ranges of $Q_{\alpha}$ and $Q_{\gamma}, 2 / 3 \leq Q_{x s s} \leq 3 / 2$. Further, its average value, $E\left\{Q_{x s s}\right\}=\int_{2}^{3} \int_{2}^{3} Q_{\alpha} / Q_{\gamma} d Q_{\alpha} d Q_{\gamma}=(5 / 2) \ln (3 / 2) \approx 1.01$, assuming that $Q_{\alpha}$ and $Q_{\gamma}$ varied independently. Similarly, for the same assumptions, the standard deviation $\sigma_{Q x s s}=$ $\sqrt{E\left\{Q_{x s s}^{2}\right\}-\left(E\left\{Q_{x s s}\right\}\right)^{2}} \approx 0.19$. In both the circuits, therefore, we observed that the mean was close to one even though the means of the temperature coefficients of the underlying parameters were not. This temperature robustness effect corresponded to the matching situation where the temperature dependencies scaled similarly and there was a net cancellation. We noted that the matching temperature robustness principle is independent of the specific ranges of the $Q_{10}$ 's that were considered. These computations were repeated for higher and lower degradation rate parameters, both of which showed similar behavior (Supplementary Fig. 7, 8).

To assess the temperature dependence of the transient response, we plotted the responses before and after scaling the parameters versus each other (binned versions are shown in Fig. $4 \mathrm{c}, \mathrm{f})$. These responses were normalized to their respective steady states. If the response did not change with temperature, the plot of the responses against each other would lie on the diagonal. As a reference, we plotted the exponential responses with time constants that are half, double, and triple, which corresponded to a slowing down of the response by twofold, a speeding up of the response by twofold, and a speeding up of the response by threefold, respectively. For the circuit without feedback, all responses were in the region spanned by the twofold and the threefold speed-up curves, which corresponded exactly to a $Q_{10}$ in the range of 2-3 for the parameter $\gamma$ (Fig. 4c). This correspondence was because the normalized response $\left(1-e^{-\gamma t}\right)$ depended only on the parameter $\gamma$, which was scaled in the range $2-3$. For the negative feedback circuit, the responses were on both sides of the twofold speed-up curve 
a.
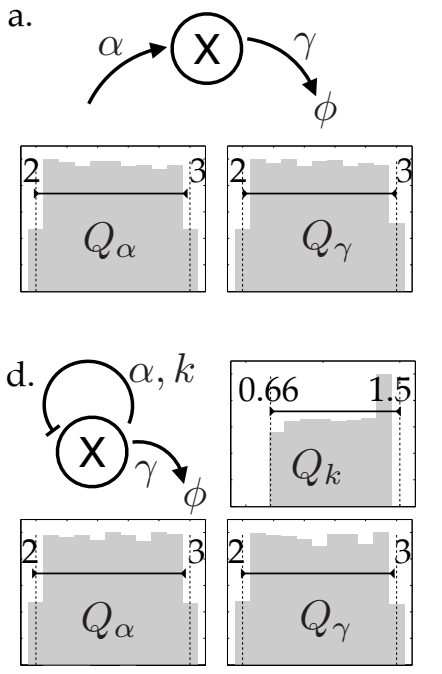

g.

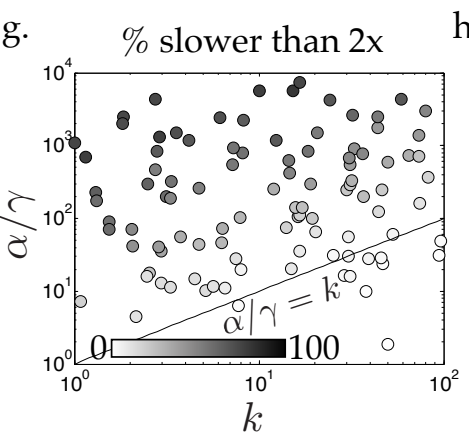

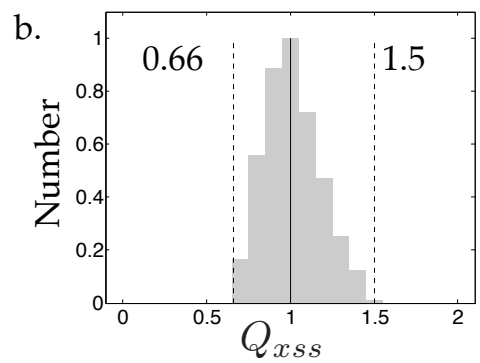

e.

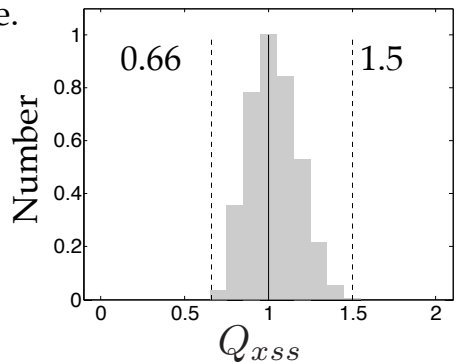

h.

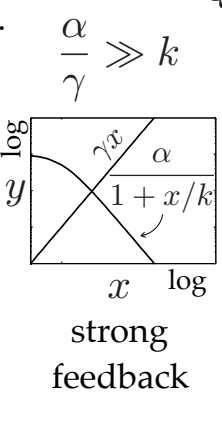

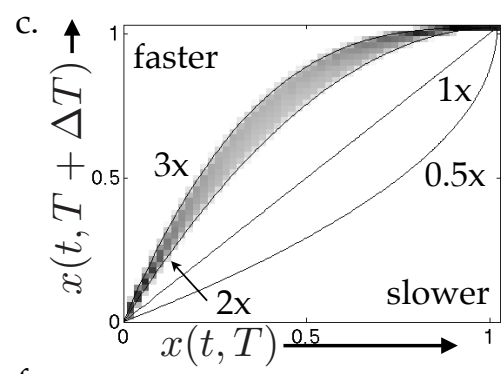

f.

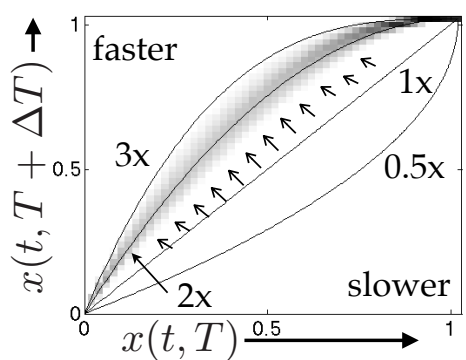

i.

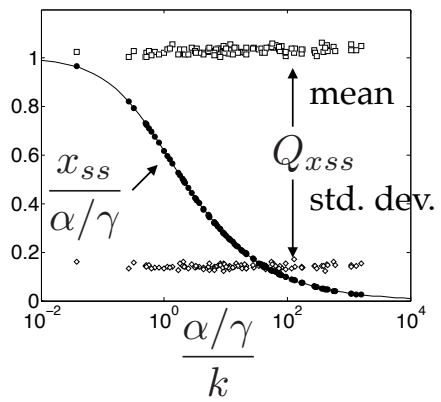

Figure 4: Strong negative feedback could facilitate the robustness of response to temperature. a. Schematic of a circuit with no feedback. Histograms show the assumed temperature coefficients of the parameters. b. Histogram of the computed temperature coefficient of the steady state. c. Normalized transient response after the temperature shift $(T \rightarrow T+\Delta T)$ is plotted versus the initial transient response. Time $(t)$ is the implicit parameter. Grayscale indicates the sum of all the trajectories at a particular grid location. Solid lines represent the curves corresponding to an exponential response and responses that are identical, faster, or slower. d. Same as in a., but for a negative feedback circuit. e. Same as in b., but for a negative feedback circuit. f. Same as in c., but for a negative feedback circuit. Arrows highlight the portion of the plot that is different from that in c. g. Dots are color-coded based on the fraction of realizations that are less than twofold faster, as estimated from f. These are plotted in the space of the parameter combinations $\alpha / \gamma$ and $k$. Solid line $\alpha / \gamma=k$ is a guide. $\mathrm{h}$. Interpretation of a weak and a strong negative feedback regime based on the relative values of the parameter combinations $\alpha / \gamma$ and $k$. i. Black solid circles represent the steady state values normalized by $\alpha / \gamma$ as a function of the negative feedback strength $\alpha / \gamma / k$. Black solid line is the analytical calculation of the same. White squares and diamonds represent the means and the standard deviations of the temperature coefficients of the steady state as a function of the negative feedback strength $\alpha / \gamma / k$, respectively. 
(Fig. 4f), suggesting that there were parameter regimes that could facilitate robustness to temperature.

We further investigated the region of the plot of the negative feedback circuit that was slower than the twofold speed-up curve. We found that for all the parameter sets, there were some scalings that were less than twofold faster than the initial response. When we counted the fraction of these scalings, we found that a larger fraction occurred for the parameter sets where $\alpha / \gamma$ was large and $k$ was small (Fig. 4g). Since $\alpha / \gamma$ is the maximum allowable level of $x$, this corresponded to a region in parameter space where the negative feedback interaction could be active (Fig. 4h). This was the region that enhanced the robustness to temperature. We checked whether this region affected the temperature dependence of the steady state and found that the mean and the standard deviation of the steady state temperature coefficient $\left(Q_{x s s}\right)$ did not change as a function of the parameter combination $\alpha / \gamma k$ (Fig. 4i). However, the absolute value of the steady state relative to the maximum allowable value $(\alpha / \gamma)$ decreased as the parameter combination $\alpha / \gamma k$ was increased. This suggested that there could be a performance cost of low dynamic range to temperature robustness, in terms of a lower steady state value, for circuits operating in the strong negative feedback regime.

We repeated this analysis for the incoherent feedforward loop circuit (Fig. 5). We considered the standard model ${ }^{18}$,

$$
\begin{aligned}
& \frac{d x}{d t}=\alpha_{x} u-\gamma_{x} x \\
& \frac{d y}{d t}=\alpha_{y} u \frac{K}{x}-\gamma_{y} y
\end{aligned}
$$

where $u$ is the input, $y$ is the level of the output protein $(Y)$, and $x$ is the level of the intermediatory protein $(\mathrm{X})$ that is activated by $u$, like $\mathrm{Y}$, and represses $\mathrm{Y}$. The parameters $\alpha_{x}$ and $\alpha_{y}$ represent the production rates of $x$ and $y$, respectively. Similarly, the parameters $\gamma_{x}$ and $\gamma_{y}$ represent the degradation rate constants of $x$ and $y$, respectively. $K$ is the DNA 
binding constant of $\mathrm{X}$ to the promoter of $\mathrm{Y}$. The repression function is approximated, $K /(x+$ $K) \approx K / x$. The nominal values of the parameters were chosen as follows: $\alpha_{x}=100 \mathrm{nM} / \mathrm{hr}$, $K=10 \mathrm{nM}, \gamma_{x}=1 / \mathrm{hr}, \alpha_{y}=100 \mathrm{nM} / \mathrm{hr}, \gamma_{y}=1 / \mathrm{hr}$, and the input,

$$
u= \begin{cases}1, & \text { if } t<0 \\ 2, & \text { if } t \geq 0\end{cases}
$$

These parameters were similar to those discussed previously ${ }^{18}$ and above. The input $u$ scales the net production rate of $x$ and $y$ in the indicated fashion.
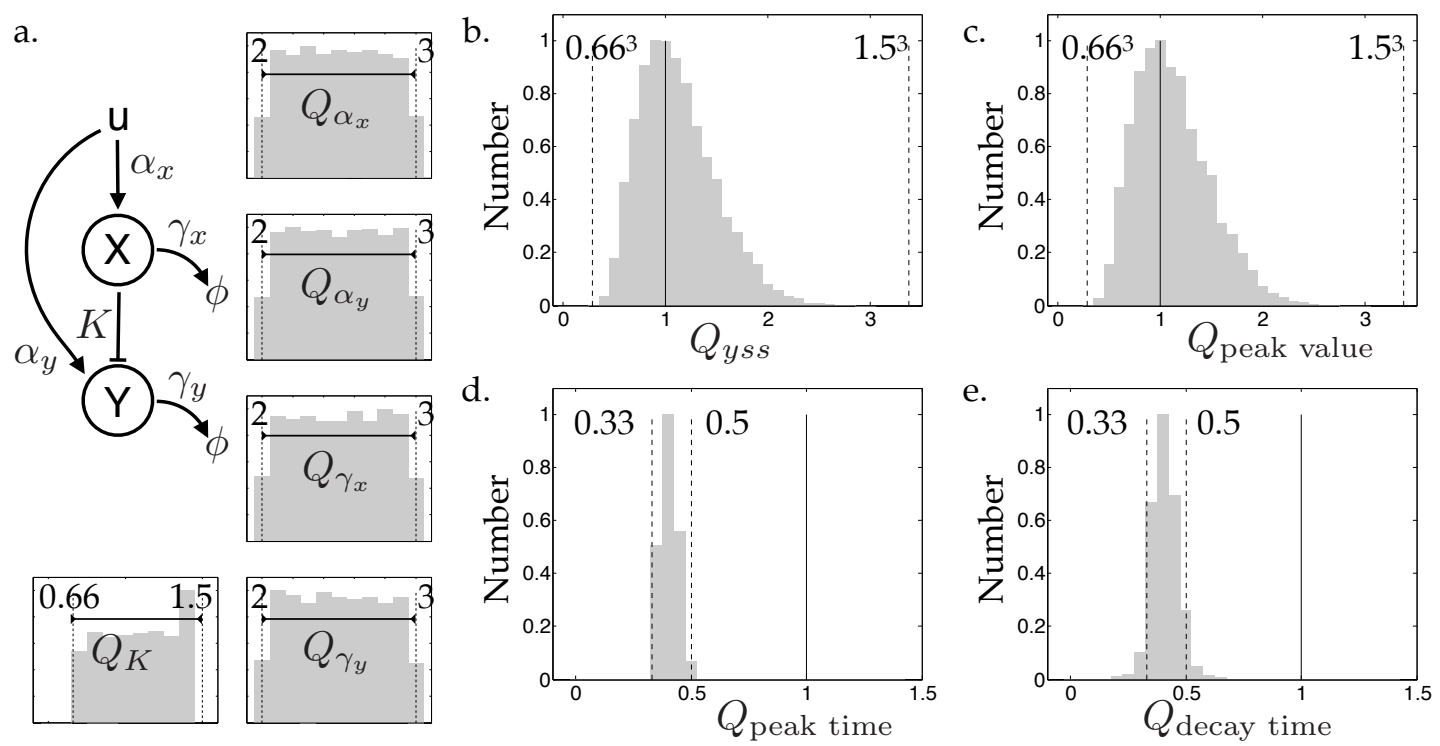

Figure 5: Computational assessment of temperature dependence of a feedforward loop. a. Schematic of a feedforward loop. Histograms show the assumed temperature coefficients of the parameters in the computations. Histograms of the computed temperature coefficient of $b$. the steady state, $c$. the peak value of the transient response, d. the peak time of the transient response, and e. the decay time of the transient response.

We assessed the temperature dependence of the steady state and transient response of $y$ due to the temperature dependence of the parameters, modeled using the temperature coefficient $Q_{10}$. The $Q_{10}$ 's of $\alpha_{x}, \gamma_{x}, \alpha_{y}$, and $\gamma_{y}$ were taken in the range 2-3 and the $Q_{10}$ of $K$ was taken in the range $0.66-1.5$ (Fig. 5a.).

The distribution of the temperature coefficients of the steady state had a mean of 1.11 
Generally, if the sets of parameters $\alpha_{x}, \alpha_{y}$ and $\gamma_{x}, \gamma_{y}$ had matching temperature dependences, which is likely due to the similar nature of processes lumped as these parameters, then we might get a temperature compensatory effect.

We assessed the temperature dependence of the transient response by considering three metrics - the peak amplitude, the time taken to rise to the peak amplitude, and the time taken to decay from the peak amplitude to $5 \%$ of its final value. The histograms of the temperature coefficient of each of these are shown in Fig. 5c-e. The peak amplitude of the transient response had a largely similar histogram as that of the steady state, with a mean of 1.12 and a standard deviation of 0.36 . The histograms of the rise time and the decay time had a mean of 0.40 and 0.41 , respectively. Both of these histograms had values predominantly in the range between $1 / 3$ and $1 / 2$. This suggested the dominant effect of the degradation rate parameters because the timescale properties, which vary as the inverse of the degradation rate parameters, have temperature coefficients in the range $1 / 3-1 / 2$. The conclusions were similar when we repeated these computations for different degradation rate parameters (Supplementary Fig. 9, 10) as well as without the above approximation in the production term of $y$ (Supplementary Fig. 11). A similar approach could also be used for other circuits, such as a coherent feedforward loop (Supplementary Fig. 12, 13). 


\section{Discussion of the experimental measurements in the context of computations.}

Finally, we placed the experimental measurements in the context of the computational assessment.

We directly estimated the growth rate parameter from the data (Supplementary Fig. 14). The growth rate is the dominant component of the parameter $\gamma$ for stable proteins. We noted that this rate changed as a function of the growth stage of the cells. Further, the peak growth rate changed in the range between onefold and twofold with a change in the temperature from $29^{\circ} \mathrm{C}$ to $37^{\circ} \mathrm{C}$. This was in a similar range as that of the $Q_{10}$ value chosen for this parameter.

The computations for the negative feedback circuit suggested that in addition to the matched temperature dependencies, certain parameter regimes could also facilitate temperature robustness but at a performance cost of low dynamic range, similar to the observations in a two-state model ${ }^{11}$. Experimental measurements seemed to be consistent with this (Fig. 2 ), as the change in the trajectories when the temperature was increased from $29{ }^{\circ} \mathrm{C}$ to 37 ${ }^{\circ} \mathrm{C}$ was smaller for low aTc (strong feedback) in comparison to high aTc (weak feedback) and the expression levels were also lower for the strong feedback. However, two aspects of the experimental conditions were not in the numerical computations. The first aspect was the possible temperature dependence of the GFP properties, such as its brightness and maturation dynamics, which might be different for different temperatures. The second aspect was the use of aTc levels to access different parameter regimes as the binding of aTc to TetR might also depend on temperature, implying a comparison of circuits with different effective DNA binding affinities of TetR. We also plotted the transient responses against each other for the strong and the weak negative feedbacks, as in Fig. 2c, f, but no significant conclusion could be made (Supplementary Fig. 15), perhaps due to the low initial brightness.

We have previously noted the effect of temperature on a similar negative feedback loop in a cell-free context ${ }^{19}$. Based on the analysis presented here, we revisited these data to assess whether a stronger negative feedback could have a better temperature robustness 

The data were of the response of the negative feedback circuit, at two different aTc levels corresponding to a strong and a weak feedback, and a constitutive promoter in a cell-free context, measured at four different temperatures $-26{ }^{\circ} \mathrm{C}, 29{ }^{\circ} \mathrm{C}, 33{ }^{\circ} \mathrm{C}$, and $37{ }^{\circ} \mathrm{C}$. (Fig. $6 \mathrm{a}-\mathrm{c}$; see the Methods section). We plotted the time taken to reach half the final value, expecting that the strong negative feedback would minimize the change in this quantity with temperature. We observed this in both instances (Fig. 6d), when comparing the strong feedback to the weak feedback and when comparing the strong feedback to the constitutive promoter (no feedback), providing experimental evidence for the temperature robustness effect of negative feedback. Next, we plotted the trajectories for the strong negative feedback, the weak negative feedback, and the constitutive promoter against each other (Fig. 6e, f) in order to compare with the computational expectation that the trajectories for the strong negative feedback would be closer to each other in comparison to the other two cases (Fig. 2c, f). We found evidence of this when we compared the responses at $26{ }^{\circ} \mathrm{C}$ to those at 33 ${ }^{\circ} \mathrm{C}$ or at $37^{\circ} \mathrm{C}$. The responses of the constitutive promoter and the weak negative feedback were similar to each other. Overall, these measurements provided experimental evidence regarding the temperature robustness effect of negative feedback.

From the feedforward loop computations, we noted that the dominant temperaturedependent effect on the peak time was due to the degradation-related parameters (Fig. $5 \mathrm{~d})$. We found that this was consistent with the experimental measurements, as the $\approx 72 \%$ decrease in the peak time as the temperature increased from $29{ }^{\circ} \mathrm{C}$ to $37^{\circ} \mathrm{C}$ (Fig. 3b) correlated with the $\approx 72 \%$ increase in the cell growth rate (assessed from the time to reach $\mathrm{OD}=0.5$, Supplementary Fig. 4), which functioned like the degradation parameter in the feedforward model for the protein X.

We investigated a possible robustness versus performance tradeoff in the feedforward loop model, similar to the tradeoff noted for the negative feedback loop. A key performance attribute of the feedforward loop model is the exact adaptation that arises in the model 
a.

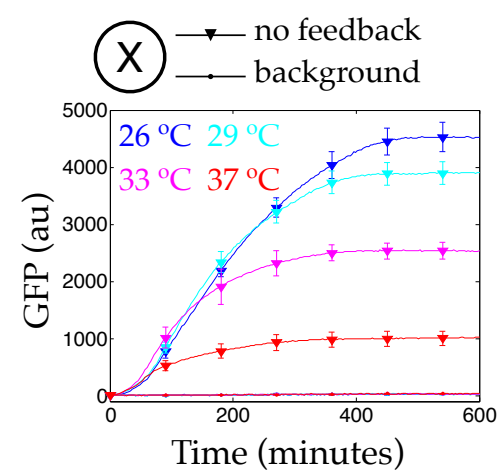

d.

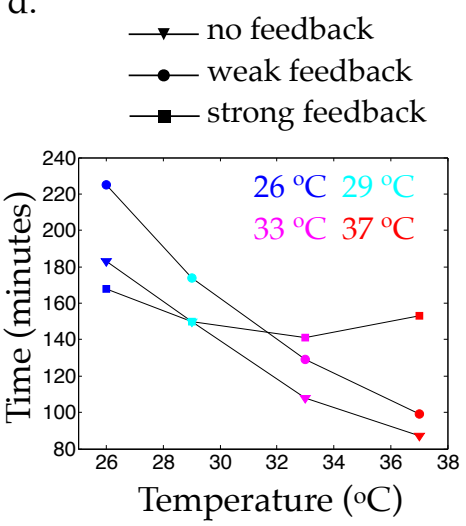

b.
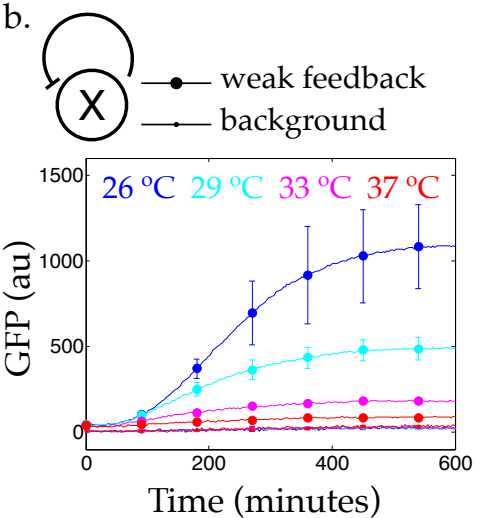

e.

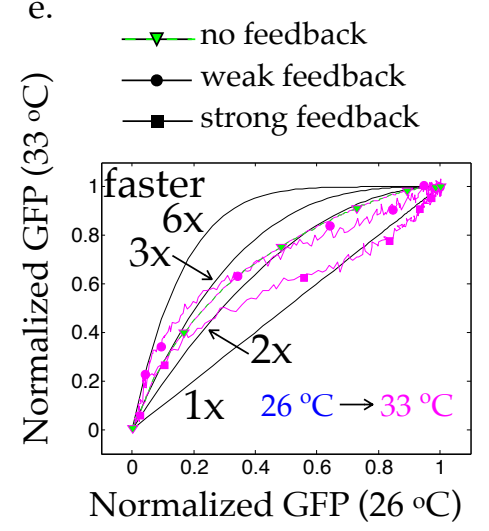

c.
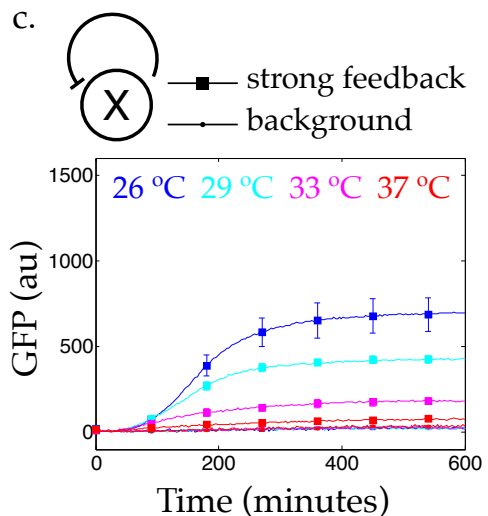

f.

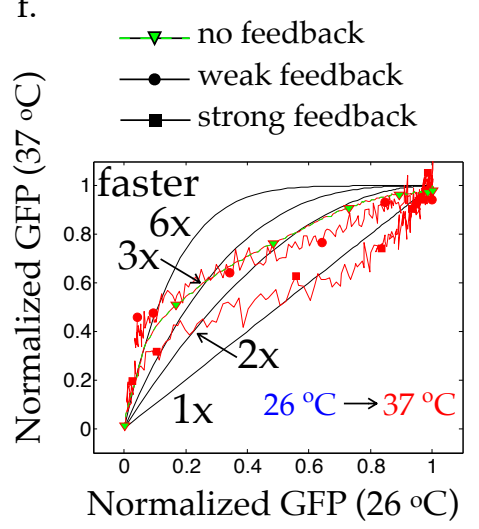

Figure 6: Experimental evidence for temperature robustness effect of strong negative feedback in a cell-free context. Trajectories for a. the constitutive promoter (no feedback), b. the weak negative feedback loop, and c. the strong negative feedback loop at $26{ }^{\circ} \mathrm{C}$ (blue line), $29{ }^{\circ} \mathrm{C}$ (cyan line), $33{ }^{\circ} \mathrm{C}$ (magenta line), and $37{ }^{\circ} \mathrm{C}$ (red line). Error bars represent the standard deviations of three independent measurements. d. Time taken to reach half the final value for the above conditions. e. Response at $33{ }^{\circ} \mathrm{C}$ normalized by its final value versus the response at $26{ }^{\circ} \mathrm{C}$ normalized by its final value for the above conditions. Black lines represent guides for when an exponential response speeds up by the indicated numbers. Green color additionally marks the line and the symbols for the no feedback case. f. Same as e., except that the response on the y-axis is at $37^{\circ} \mathrm{C}$. 

only on the parameters and not on the input, $y_{s s}=K \frac{\alpha_{y} \gamma_{x}}{\alpha_{x} \gamma_{y}}$. In the other limit of this approximation $x \ll K$, there is no adaptation, and the steady state output depends on the input $y_{s s}=u \frac{\alpha_{y}}{\gamma_{y}}$. We noted that the worst-case $Q_{y s s}$ range was larger with adaptation $\left(\left[0.66^{3}, 1.5^{3}\right]\right)$ than without adaptation $([0.66,1.5])$. This could be a possible tradeoff in performance (adaptation) and robustness to temperature (worst-case $Q_{y s s}$ range). This analysis assumed that the input did not depend on temperature. If the input depended on temperature, a parameter regime $\left(u \gg K_{u}\right)$ in the expanded model (Supplementary Fig. 11) could weaken the effect of the temperature dependence of the input on the temperature dependence of the steady state output. In this regime, the performance was further degraded in the sense that the dependence of steady state output on the input was weaker. This analysis suggested that robustness to temperature at a performance cost through the choice of parameter regimes might also occur in a feedforward loop.

Summary. Assessing and designing robustness to temperature in biomolecular circuits is an important challenge for possible applications. For a synthetic oscillator, the temperature dependence of the period was measured, modeled, and modified to be relatively robust to temperature using matched temperature dependencies ${ }^{9,10}$. For simple synthetic networks in yeast (a positive feedback loop and a negative feedback loop), the temperature dependence was experimentally measured and its origins were investigated ${ }^{13}$. We addressed this using experimental measurements and mathematical models of two biomolecular circuit motifs negative feedback and feedforward loop - in E. coli. We found that the overall response of their circuit realizations changed with temperature, both in the amplitude and the transient response. We analyzed the mathematical models of these circuits, and highlighted that in addition to having parameters with matched temperature dependencies, certain parameter regimes could also facilitate temperature robustness, albeit at a performance cost. We discussed the experimental measurements of the negative feedback loop in the context of the role of the parameter regimes in facilitating temperature robustness. These results should 
contribute to building a framework for assessing and designing temperature robustness in biomolecular circuits.

There are four pillars that have been proposed for understanding the temperature dependence of biomolecular circuits ${ }^{13}$. These are - the cell fate choice between growth and stress resistance, the diminished growth of a subtype of cells, the Arrhenius dependence of the reaction rates, and the altered protein conformational dynamics. The significance of the first two pillars in the circuits presented here would require single cell measurements. This is an important direction of future work. We noted that cells entered the stationary phase at different times for different temperatures (Supplementary Fig. 1, 4), and this might be the reason for some of the observed temperature dependence. The Arrhenius dependence of reaction rates has experimental utility ${ }^{9,10}$ and is a widely used assumption in modeling work $^{11}$ as well. Here, the temperature dependence of a reaction rate parameter is modeled as $k=k_{0} \exp \left(-\Delta E / k_{B} T\right)$, where $k_{0}$ is a constant, $\Delta E$ is an activation energy, $k_{B}$ is the Boltzmann constant, and $T$ is the absolute temperature. In fact, the $Q_{10}$ framework used in this study may be understood as an instance of the Arrhenius temperature dependence for a specific value of $\Delta E$ and a choice of the temperature range. Finally, altered protein conformational dynamics is another important factor that determines the temperature dependence of biomolecular circuits. The effects of these dynamics may be modeled coarsely; for exam-

ple, through the lumped parameters for DNA binding as was done here, or more completely through molecular dynamics simulations. The latter scale of models are important in creating a platform to computationally predict the temperature dependence of biomolecular circuits.

\section{Materials and Methods}

The plasmids and bacterial strains as well as the methods of experimental measurements, data analysis, and computations used in this research are described below. 
Plasmids and Bacterial Strains. The negative feedback loop was obtained from Prof. M. B. Elowitz ${ }^{14}$. The plasmid is $\mathrm{pZS}^{*} 21$ tetR-egfp and is in the E. coli strain DH5 $\alpha$. The feedforward loop pBEST-OR2-OR1-Pr-araC, pBAD-TetR, pBAD-TetO1-deGFPssrA is a plasmid from the laboratory of Prof. Richard M. Murray (Addgene plasmid \# 45789; http://n2t.net/addgene:45789; RRID:Addgene_45789). This was transformed into the E. coli strain MG1655.

Measurements. For the negative feedback loop experiment, two strains — one containing the plasmid and the other without any plasmid — were grown overnight in minimal M9CA media (1x M9 salts, 1.0\% glucose, 0.1\% Casamino Acids, $0.5 \mu \mathrm{g} / \mathrm{ml}$ Thiamine, $0.2 \mathrm{mM}$ $\mathrm{MgSO}_{4}, 0.1 \mathrm{mM} \mathrm{CaCl}$, $\mathrm{pH} 7.0$, from Teknova) at the required temperature $\left(29{ }^{\circ} \mathrm{C}\right.$ or 37 $\left.{ }^{\circ} \mathrm{C}\right)$. These were diluted 1:100 in fresh media. The culture with the plasmid was grown in the presence of the antibiotic Kanamycin $(50 \mathrm{ng} / \mathrm{ml}) .190 \mu \mathrm{l}$ of each strain and of the media was placed in the wells of a 96-well plate (Perkin Elmer). The following final concentrations of the inducer aTc (in $\mu \mathrm{g} / \mathrm{ml}$ ) were added in three wells of the negative feedback strain to make a total volume of $200 \mu \mathrm{l}: 100,20,4$. In the wells of the strain without any plasmid and of the media, $10 \mu \mathrm{l}$ of a 1:1000 dilution of ethanol in media was added to make a total volume of $200 \mu \mathrm{l}$. Each sample was placed in triplicate. The plate was incubated in a plate reader (Biotek Synergy H1) for 19 hours with shaking (double orbital, continuous, 237 cpm) at the required temperature. Every 10 minutes, the fluorescence $(\mathrm{ex} / \mathrm{em}=485 / 530 \mathrm{~nm}$, gain 61 ) and the optical density (absorbance at $600 \mathrm{~nm}$ ) were measured. The measurements of the well containing only the media provided the background for these measurements. These measurements were repeated on different days for the temperatures of $29^{\circ} \mathrm{C}(\mathrm{N}=4$ days $)$ and $37^{\circ} \mathrm{C}(\mathrm{N}=3$ days $)$.

The feedforward loop strain was grown overnight in LB media supplemented with antibiotic Ampicillin $(100 \mu \mathrm{g} / \mathrm{ml})$ at $37^{\circ} \mathrm{C}$. It was subsequently diluted in minimal media with 
the antibiotic and incubated with the inducer aTc $(1 \mathrm{ng} / \mathrm{ml})$ for two hours. Next, $0.2 \%$ of the inducer Arabinose was added to the incubated culture and $200 \mu \mathrm{l}$ of it was placed in a 96-well plate (Eppendorf). The plate was placed in a plate reader (Biotek Synergy H1) and incubated at the required temperature. The incubation was for 10 hours at $37{ }^{\circ} \mathrm{C}$ and for 16 hours at $29{ }^{\circ} \mathrm{C}$. The fluorescence $(\mathrm{ex} / \mathrm{em}=485 / 525 \mathrm{~nm}$ ) and the optical density (absorbance at $600 \mathrm{~nm}$ ) of each culture were measured every 5 minutes, with a 2-minute shaking in between the readings. The samples were placed in duplicate and the above protocol was repeated for $\mathrm{N}=3$ days at each temperature.

Data Analysis. All the data analysis was performed in MATLAB.

For the negative feedback loop, the fluorescence and the optical density of all the samples across all days were used to get a sample mean and a sample standard deviation. The mean of the media background (fluorescence and optical density) was subtracted from the sample means. In these traces, there was an initial period where the background and the samples overlapped in the sense of the error bars given by the respective standard deviations. Therefore, for each sample, the time after which there was no overlap was considered. This helped to avoid the divide-by-zero (or a small number) situation and the associated large fluctuations in the optical density-normalized fluorescence. The optical density and the fluorescence data are shown in Supplementary Fig. 1. The standard deviations of the optical density and fluorescence were obtained from the day-to-day samples. The optical density-normalized fluorescence values are shown in Supplementary Fig. 2. The standard deviation of the optical density-normalized fluorescence were obtained from these via quadrature, assuming that they were uncorrelated. The data at $29^{\circ} \mathrm{C}$ showed a substantial variation in comparison to the data at $37{ }^{\circ} \mathrm{C}$, which could be because the temperature controller of the plate reader was more uniform at $37{ }^{\circ} \mathrm{C}$ (Suppplementary Fig. 3). As the temperature profiles of the first two repeats looked similar, these two readings were considered for the data at $29{ }^{\circ} \mathrm{C}$, as shown in Fig. 2b-d. 


\begin{abstract}
Numerical Computations. Ordinary differential equations were solved in MATLAB using
\end{abstract} the function ode23s.

Cell-free Experiments. These experiments were performed in a cell-free transcription translation system ${ }^{20,21}$. The negative transcriptional feedback circuit used for the experiment contained the transcriptional repressor TetR expressed from a self-repressible promoter (Addgene plasmid \# 45774 ; http://n2t.net/addgene:45774; RRID:Addgene_45774). TetR was fused to the green fluorescent protein variant deGFP. The constitutive promoter used was the lambda repressor Cro promoter (OR2-OR1- $\left.P_{r}\right)$. It drove the expression of deGFP and was a positive control for the cell-free reactions. These measurements were performed in a plate reader (BioTek Synergy H1), with measurement intervals set at 3 minutes for a total duration of 10 hours. The fluorescence was measured using excitation and emission at the wavelengths $485 \mathrm{~nm}$ and $525 \mathrm{~nm}$, respectively. The total reaction volume was $10 \mu$ l. The concentration of the negative feedback plasmid used in each reaction was $2 \mathrm{nM}$. These were then repeated at the different desired temperatures. The aTc concentration of $0.5 \mu \mathrm{g} / \mathrm{ml}$ corresponded to the relatively stronger negative feedback while the $5 \mu \mathrm{g} / \mathrm{ml}$ aTc concentration corresponded to the relatively weaker negative feedback. This concentration of the plasmid was chosen so that the reaction was in the linear range, in the sense that doubling or halving the concentration would approximately double or halve the response, respectively (Supplementary Fig. 16, aTc $=5 \mu \mathrm{g} / \mathrm{ml}$ ). The individual traces for the data presented in Fig. 6 are shown in Supplementary Fig. 17. A smoothened version of the plots in Fig. 6e, $\mathrm{f}$ is shown in Supplementary Fig. 18. The models considered in the main text 
did not apply directly to cell-free systems as there was no active degradation in the cell-free system. A model for the cell-free system that included the resource limitation was considered to computationally assess the dependence of the transient response on temperature (Supplementary Fig. 19).

\section{Acknowledgement}

We thank C. A. Hayes for her help with the cell-free experiments. We are grateful to the referees for their valuable comments. A. Patel acknowledges financial support from a DeitY Fellowship. This work was supported in part by Science and Engineering Research Board grant no. SB/FTP/ETA-0152/2013.

\section{Supporting Information Available}

- Supplementary Figures: (A) Supplementary Experimental Data, (B) Steady State of the Negative Feedback Model, (C) Flowchart for Computing $Q_{x s s}$, (D) Computational Assessment of Model Variants, (E) Computational Assessment of a Coherent Feedforward Loop Model, (F) Additional Data Analysis of Negative Feedback Loop Experiments, (G) Additional Data, Data Analysis, and Model for the Cell-Free Experiments.

This material is available free of charge via the Internet at http://pubs.acs.org/.

\section{References}

1. Razavi, B. (2016) The Bandgap Reference [A circuit for all seasons]. IEEE Solid-State Circuits Mag. 8, 9-12.

2. Hu, J., Huang, H., Bai, M., Zhan, T., Yang, Z., Yu, Y., and Qu, B. (2017) A high 
sensitive fiber-optic strain sensor with tunable temperature sensitivity for temperaturecompensation measurement. Sci. Rep. 7.

3. Cardinale, S., and Arkin, A. P. (2012) Contextualizing context for synthetic biologyidentifying causes of failure of synthetic biological systems. Biotechnol. J. $\underline{7}, 856-866$.

4. Karamasioti, E., Lormeau, C., and Stelling, J. (2017) Computational design of biological circuits: putting parts into context. Mol. Syst. Des. Eng. 2, 410-421.

5. Nakajima, M., Imai, K., Ito, H., Nishiwaki, T., Murayama, Y., Iwasaki, H., Oyama, T., and Kondo, T. (2005) Reconstitution of circadian oscillation of cyanobacterial KaiC phosphorylation in vitro. Science $308,414-5$.

6. Reyes, A. B., Pendergast, J. S., and Yamazaki, S. (2008) Mammalian peripheral circadian oscillators are temperature compensated. J. Biol. Rhythms 23, 95-98.

7. Oleksiuk, O., Jakovljevic, V., Vladimirov, N., Carvalho, R., Paster, E., Ryu, W. S., Meir, Y., Wingreen, N. S., Kollmann, M., and Sourjik, V. (2011) Thermal robustness of signaling in bacterial chemotaxis. Cell 145, 312-21.

8. Ruoff, P., Zakhartsev, M., and Westerhoff, H. V. (2007) Temperature compensation through systems biology: Temperature compensation of fluxes. The FEBS Journal 274, 940-950.

9. Stricker, J., Cookson, S., Bennett, M. R., Mather, W. H., Tsimring, L. S., and Hasty, J. (2008) A fast, robust and tunable synthetic gene oscillator. Nature 456, 516-519.

10. Hussain, F., Gupta, C., Hirning, A. J., Ott, W., Matthews, K. S., Josic, K., and Bennett, M. R. (2014) Engineered temperature compensation in a synthetic genetic clock. Proc. Natl. Acad. Sci. U.S.A. 111, 972-977.

11. Sen, S., and Murray, R. M. Temperature dependence of biomolecular circuit designs. 52nd IEEE Conference on Decision and Control. 2013; pp 1398-1403. 
12. Sen, S., Kim, J., and Murray, R. M. Designing robustness to temperature in a feedforward loop circuit. 53rd IEEE Conference on Decision and Control. 2014; pp 4629-4634.

13. Charlebois, D. A., Hauser, K., Marshall, S., and Balázsi, G. (2018) Multiscale effects of heating and cooling on genes and gene networks. Proc. Natl. Acad. Sci. U.S.A. 115, E10797-E10806.

14. Rosenfeld, N., Elowitz, M. B., and Alon, U. (2002) Negative autoregulation speeds the response times of transcription networks. J. Mol. Biol. 323, 785-93.

15. Shen-Orr, S. S., Milo, R., Mangan, S., and Alon, U. (2002) Network motifs in the transcriptional regulation network of Escherichia coli. Nat. Genet. 31, 64-68.

16. Lodish, H. F., Ed. Molecular cell biology, 4th ed.; W.H. Freeman: New York, 2000.

17. Elowitz, M. B., and Leibler, S. (2000) A synthetic oscillatory network of transcriptional regulators. Nature $\underline{403,335-338 .}$

18. Goentoro, L., Shoval, O., Kirschner, M. W., and Alon, U. (2009) The Incoherent Feedforward Loop can provide Fold-Change Detection in gene regulation. Mol. Cell 36, 894-899.

19. Sen, S., and Murray, R. M. (2014) Negative feedback facilitates temperature robustness in biomolecular circuit dynamics. bioRxiv doi: 10.1101/007385.

20. Sun, Z. Z., Hayes, C. A., Shin, J., Caschera, F., Murray, R. M., and Noireaux, V. (2013) Protocols for implementing an Escherichia coli based TX-TL cell-free expression system for synthetic biology. J. Vis. Exp. e50762.

21. Shin, J., and Noireaux, V. (2012) An E. coli cell-free expression toolbox: application to synthetic gene circuits and artificial cells. ACS Synth. Biol. 1, 29-41. 


\section{Graphical TOC Entry}

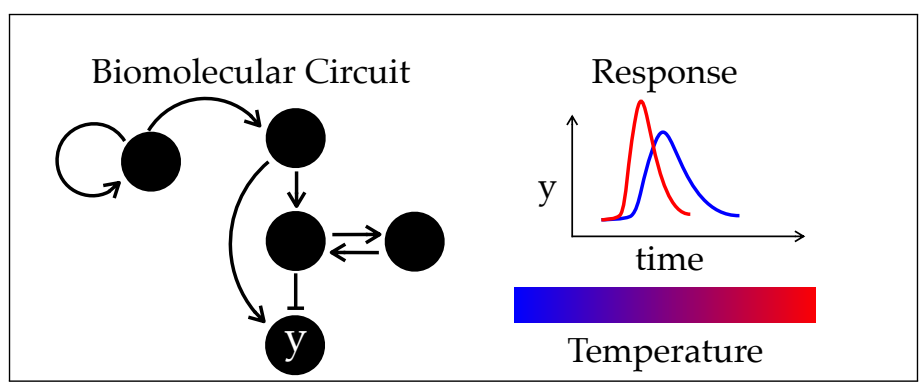

